\title{
Diagnosis and management of a wooden orbital foreign body: case report
}

\author{
JOHN A. MACRAE \\ From the Department of Ophthalmology, Ninewells Hospital and Medical School, \\ Dundee DD2 IUB, Scotland
}

SUMMARY The case history of a patient with a retained wooden orbital foreign body is presented. The report illustrates the diagnostic limitations of orbital CAT scanning and ultrasonography. Systemic corticosteroid therapy facilitated the localisation and surgical removal of the foreign body.

A wooden orbital foreign body may present considerable diagnostic difficulty, as illustrated by the reports of Von Márton (1969), Ferguson (1970), and Legras and Lecoq (1970). The radiolucent nature of the foreign material prevents localisation by routine orbital radiography, but with the advent of orbital CAT scanning and ultrasonography the likelihood of identification should be increased.

The report which follows demonstrates the diagnostic and therapeutic problems still presented by such cases despite orbital investigations by CAT and ultrasonography.

\section{Case report}

A 46-year-old man attended the local casualty department after sustaining an injury to his left eye. While pruning a tree he had received a glancing blow from the terminal twig of a branch. The casualty officer diagnosed a conjunctival abrasion, prescribed antibiotic ointment, and advised the patient to seek specialist advice if the eye did not settle.

During the next 3 days the patient's left eye became increasingly painful and swollen. Four days after the injury he attended the ophthalmology clinic at Ninewells Hospital.

On examination the unaided visual acuity of the left eye was found to be 6/9. There was marked chemosis of the lower bulbar conjunctiva with prolapse between the eyelids. A bulbar conjunctival laceration approximately $10 \mathrm{~mm}$ in length was noted (Fig. 1). The cornea was clear, anterior chamber quiet, pupil normally reactive, and fundus normal on direct ophthalmoscopy.

The patient was admitted to the ophthalmology department and systemic and topical antibiotic

Correspondence to Mr Macrae. therapy begun. Orbital $x$-rays did not reveal any evidence of a radioopaque foreign body or fracture. The conjunctival wound was explored, cleaned, and repaired under general anaesthesia.

Postoperatively the conjunctival chemosis did not resolve and the ocular movements of the left eye were noted to be limited. Repeated conjunctival swabs were sterile, microscopy of conjunctival

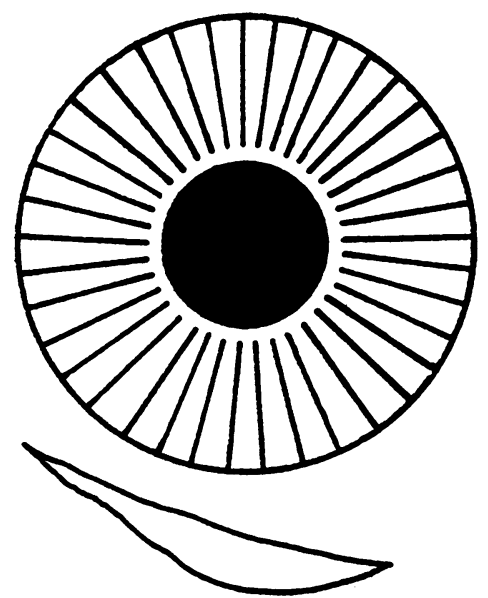

Fig. 1 Diagram of left eye showing position and extent of the conjunctival laceration

scrapings did not reveal any micro-organisms or fungal elements, and culture on Sabouraud's agar was negative.

The conjunctiva remained prolapsed, and 7 days after the surgical repair a mattress lid suture was inserted under local anaesthesia to provide protection and tamponade. The chemosis then subsided considerably and the patient was discharged from 


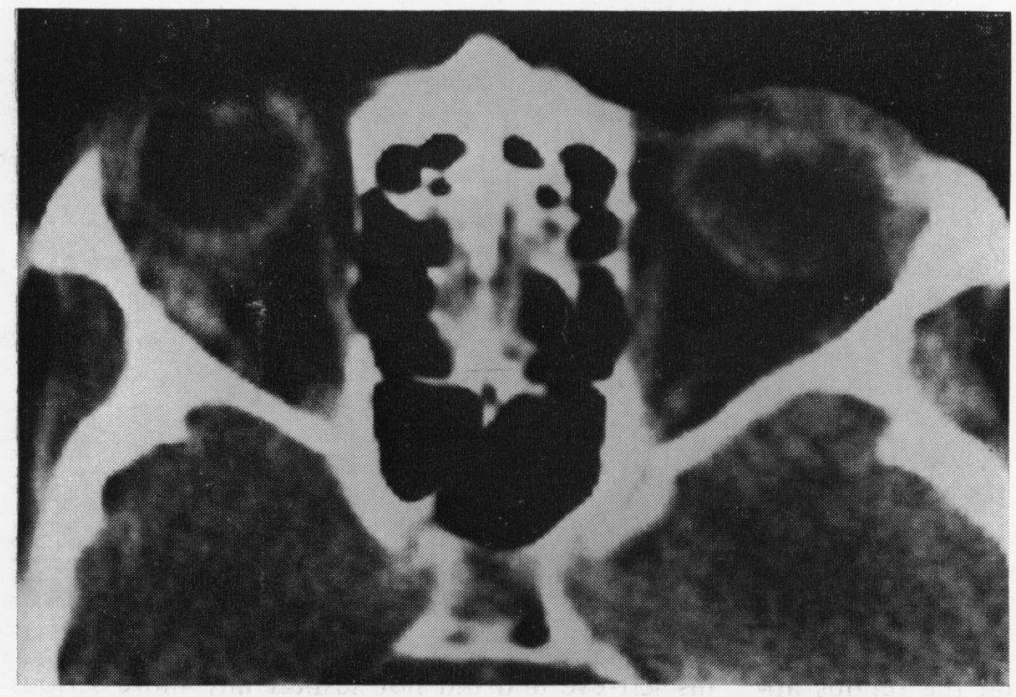

Fig. 2 Orbital CAT scan after intravenous injection of contrast medium. An area of increased density is apparent posterolaterally in the left orbit in association with the lateral rectus muscle

hospital with the lid suture in situ. Two weeks later the suture was removed and the conjunctival chemosis reappeared, particularly at the nasal side, where a pedunculated lesion developed. This lesion was excised under local anaesthesia and subjected to histological examination, which indicated a mixed acute and chronic inflammatory cellular infiltration. Special staining techniques did not reveal any microorganisms or fungi.

Diplopia then became a prominent symptom. The patient developed a left convergent strabismus of 40 prism dioptres in the primary position, with limitation of all ocular movements of the left eye, especially abduction, which was almost absent.

An orbital CAT scan was performed. This showed an area of increased density corresponding to the left lateral rectus muscle. Slight enhancement followed the injection of contrast medium, raising the possibility of a granulomatous lesion. No positive evidence of an orbital foreign body was detected (Fig. 2).

Similarly, orbital ultrasonography did not provide any definite evidence of a retained orbital foreign body.

Fundus examination of the left eye was performed by binocular indirect ophthalmoscopy. This revealed a lesion identical in appearance to a shallow radial scleral buckle, extending from the inferior temporal periphery to the posterior pole below the macular area (Fig. 3). This finding was consistent with external pressure on the sclera, possibly due to a localised granulomatous lesion or a foreign body lying in contact with the globe.

The chemosis of the lower bulbar conjunctiva persisted.
After the conjunctival biopsy and orbital CAT scan, both of which suggested a granulomatous pathology, and in the absence of an infective aetiology, it was decided to initiate a trial of corticosteroid therapy. The patient received oral prednisolone, $40 \mathrm{mg}$ daily, and topical betamethasone to the left eye, in addition to antibiotic therapy.

The conjunctival chemosis rapidly subsided, the range of movement of the left eye increased, and the fundus lesion became shallower. The visual acuity of the left eye remained 6/9. The dose of oral prednisolone was retained at the level of $40 \mathrm{mg}$ per day for 2 weeks, then gradually reduced to $20 \mathrm{mg}$ daily over a further 4-week period. By this time the

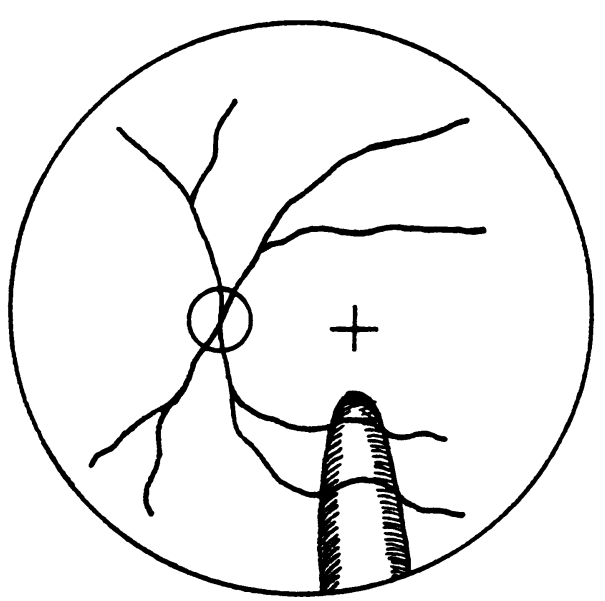

Fig. 3 Diagram showing fundus appearance of left eye with a radially situated scleral "buckle' 
Fig. 4 Removal of the wooden foreign body from the left orbit. The eye is rotated upwards and the lower eyelid retracted with a squint hook

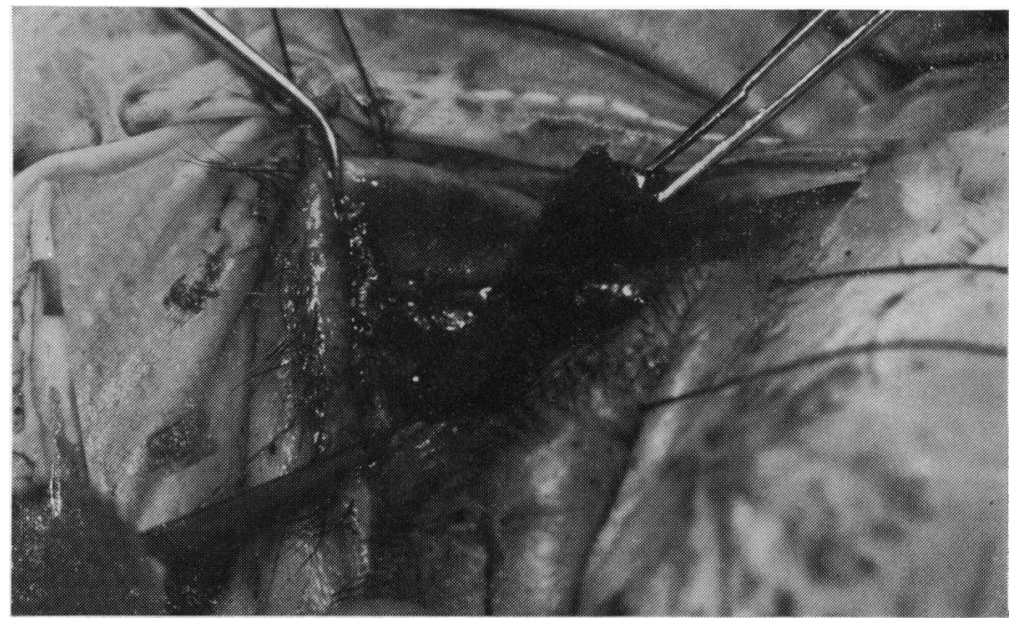

chemosis had almost resolved and the fundus appearance become normal.

With the subsidence of the tissue swelling it was then possible to palpate, through the left lower eyelid with the eye in upgaze, a circumscribed hard mass situated on the surface of the globe just lateral to the position of the inferior rectus muscle.

The patient underwent exploratory surgery under general anaesthesia. The operative findings were dramatic. Approximately $15 \mathrm{~mm}$ from the limbus at the lateral border of the inferior rectus muscle a hard circular mass was found in contact with the sclera and encapsulated by fibrous tissue. The mass was grasped with forceps and withdrawn to reveal its identity as the broken end of a wooden twig measuring $23 \times 4 \mathrm{~mm}$ (Figs. 4 and 5). The wound was sutured and a subconjunctival injection of gentamicin given. Postoperative recovery was uneventful and the patient was discharged from hospital 2 days after surgery. The systemic corticosteroid therapy was gradually reduced and was discontinued 10 days postoperatively.

The residual conjunctival chemosis and limitation of movement of the left eye subsequently resolved, and the visual acuity improved to $6 / 5$.

\section{Discussion}

The preceding case history plots the clinical course of a patient who sustained an eye injury which resulted in an undetected wooden foreign body remaining in his left orbit for a period of 14 weeks. Several factors prevented early recognition of the true nature of the orbital lesion.

The history elicited from the patient did not suggest direct penetration of the periocular tissues. He was aware of the branch merely brushing across his left eye and did not suspect any more serious injury.

The delay of 4 days before surgical repair could be carried out probably precluded any possibility of identifying such a deeply situated foreign body because of the profuse tissue swelling and closure of the subconjunctival tract along which the wooden twig

Fig. 5 The wooden twig removed from the left orbit

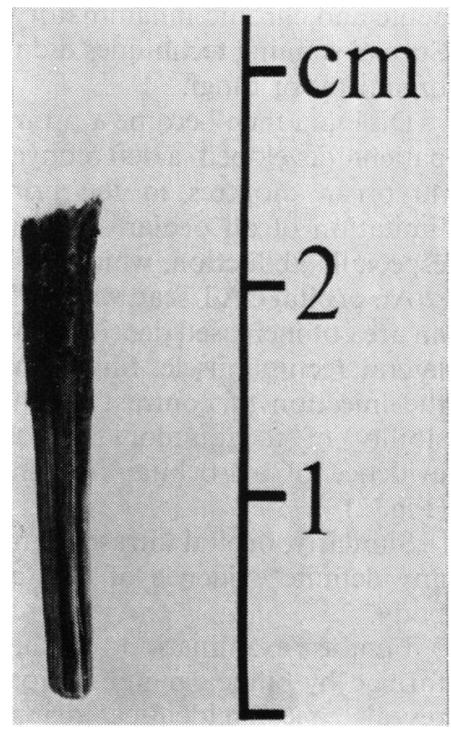

had passed. Exploration of the wound did not reveal any subconjunctival extension beyond the immediate confines of the conjunctival laceration, and apart from several particles of dirt no significant

The conjunctival chemosis was consistent with the tissue trauma, particularly in view of the delay beforeign body was apparent. 
tween the injury and wound repair. Furthermore, in the absence of proved infection, the persistence of the chemosis could have been the result of prolapse and desiccation of the conjunctiva.

Limitation of movement of the left eye could have been attributed to the mechanical restriction imposed by the bulbar conjunctival chemosis and also interference with extraocular muscle function by increased tissue tension. In retrospect, both the chemosis and interference with the movement of the left eye were the direct consequence of the granulomatous reaction induced by the wooden foreign body. Orbital radiography was unhelpful because of the radiolucent nature of the wooden foreign body.

The CAT scan was equivocal. The increased density noted in association with the left lateral rectus muscle was probably the manifestation of a granulomatous myositis, and the enhancement which followed the injection of contrast medium was in keeping with this interpretation. The foreign body was not positively identified by the CAT scan for several reasons. The foreign body lay close to the orbital floor possibly within the 'blind area' of the CAT scan described by Gawler et al. (1974). The situation of the twig, adjacent to and parallel with the inferior rectus muscle, and also the similarity in dimension and density to the muscle, would render localisation virtually impossible by standard CAT scanning techniques.

The failure of ultrasonography to localise the foreign body can also be explained in terms of its position. The close proximity of relatively dense tissues with good sound-reflecting interfaces possibly masked the foreign body to the sound wave. Furthermore, the sound emission from the ultrasonic probe would be directed towards the broken end of the twig, a structure $4 \mathrm{~mm}$ in diameter, surrounded by tissue of similar density. The foreign body would therefore possess poor sound reflecting properties and would produce a correspondingly weak echo, rendering ultrasonic diagnosis most unlikely. The case dramatically illustrates the limitations of both CAT scanning and ultrasonography in the diagnosis and localisation of a wooden foreign body situated in the orbit.

The presence and situation of a retained orbital foreign body were suggested by the fundus appearance of a scleral buckle on indirect ophthalmoscopy. However, it was the administration of corticosteroid therapy which brought about sufficient reduction in the inflammatory reaction to permit positive localisation of the anterior end of the twig by palpation through the lower eyelid. These findings provided adequate direct localising evidence to justify the surgical exploration which ultimately led to the removal of the foreign body.

The anti-inflammatory effect of corticosteroid therapy produced 2 distinct clinical benefits. Firstly, the foreign body, which had previously been masked by tissue swelling, was revealed to the palpating finger when the swelling subsided. Secondly, the surgical approach to the foreign body was rendered less hazardous by a reduction in the granulomatous reaction and conjunctival chemosis. In this particular patient the administration of systemic corticosteroids was therefore of diagnostic and therapeutic value, though the decision to start such therapy was originally made on an empirical basis.

When a patient presents with a history of an ocular injury caused by organic material such as wood, the possibility of a retained orbital foreign body must be considered, particularly when inflammatory signs persist and limitation of ocular movement is evident. The indication of a normal orbit by CAT scanning or ultrasonography does not exclude a retained wooden foreign body. However, as DukeElder (1972) stressed, it should be considered potentially hazardous to embark on a blind exploration for a suspected orbital foreign body without positive proof of its position or even of its existence. In circumstances as illustrated by the case described it is prudent to temporise until definite localising signs become apparent before surgical exploration is contemplated. Such management facilitates a planned surgical approach, thus minimising the operative trauma to the orbital structures.

I am grateful to Mrs A. Gearce for secretarial services and to Mrs A. Ellingford and Mrs R. McNeil for the preparation of photographic material.

\section{References}

Duke-Elder, S. (1972). System of Ophthalmology, Vol. 14, Part 1, pp. 666-667. Kimpton: London.

Ferguson, E. C. (1970). Deep wooden foreign bodies of the orbit. Transactions of the American Academy of Ophthalmology and Otolaryngology, 74, 778-787.

Gawler, J., Sanders, M.D., Bull, J. W. D., du Boulay, G., and Marshall, J. (1974). Computer assisted tomography in orbital disease. British Journal of Ophthalmology, 58, 571-586.

Legras, M., and Lecoq, P. J. (1970). Orbital lesions with retention of (radio-transparent) foreign bodies. Archives d'Ophtalmologie, 30, 57-60.

von Márton, D. (1969). Foreign bodies in the orbit. Ophthalmologica, 159, 49-54. 